\title{
Semi-Global 3D Line Modeling for Incremental Structure-from-Motion
}

\author{
Manuel Hofer \\ hofer@icg.tugraz.at \\ Michael Donoser \\ donoser@icg.tugraz.at \\ Horst Bischof \\ bischof@icg.tugraz.at
}

Institute for Computer Graphics and Vision

Graz University of Technology

Austria

\section{Motivation}

Recovering 3D information from a single moving camera is a widely studied field in the area of computer vision (e.g. [1]). Most of these Structurefrom-Motion (SfM) approaches are based on so-called interest points (e.g. corners) in images, which can be accurately matched using powerful descriptors like SIFT [7]. Hence the output is usually a sparse 3D point cloud along with the camera poses for all successfully integrated images. While previous methods were only able to perform pose estimation and $3 \mathrm{D}$ reconstruction in an offline way, there are now more and more incremental SfM approaches available (e.g. [4]).

Since conventional SfM approaches are based on interest points, the distribution of the obtained 3D points is usually not uniform throughout the whole reconstruction. This is due to the fact that such interest points are usually located on highly textured areas, but not on homogeneous regions or along edges. Since the result of SfM pipelines is often used as basis to generate a more dense result or for localization and navigation tasks, it would be beneficial to generate additional complementary 3D information in an efficient way. From a SfM point of view, using line segments is especially interesting for urban and indoor environments, where linear structures frequently occur. While interest points are located mostly on richly textured image locations, line segments usually mark the boundaries of objects. Hence, incorporating such features in an online SfM pipeline to create 3D line segments naturally leads to a more complete 3D representation of the underlying scene, which is beneficial for all kinds of subsequent applications.

We propose a novel approach which generates 3D line models in a semi-global way directly on-the-fly, based solely on the output of a conventional incremental SfM pipeline. The goal of our method is to generate additional complementary 3D information to improve the sparse 3D representation of the scene. In this approach, we consider the SfM pipeline as a black box and do not interfere with the pose estimation procedure. We show that 3D line reconstructions can be obtained very efficiently by using purely geometric constraints, or by additionally incorporating appearance and collinearity information. Our approach enables accurate 3D reconstruction of texture-less as well as textured man-made objects, including complex structures such as wiry objects. Figure 1 shows a reconstruction result obtained by an incremental SfM system [4], followed by a surface generation method [5], with and without the usage of additional 3D line segments obtained by our proposed method. As we can see, additional 3D information significantly improves the completeness and overall appearance of the resulting reconstructions. For more technical details, we kindly refer to the full paper.

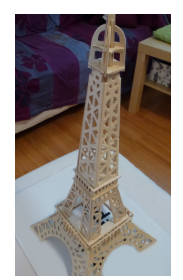

(a)

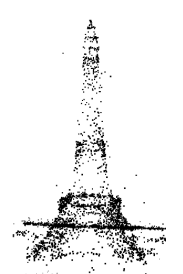

(b)

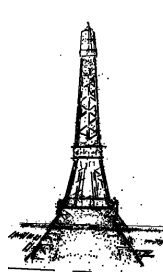

(c)

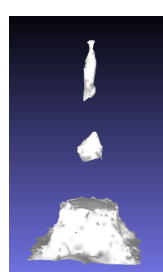

(d)

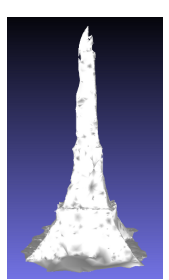

(e)
Figure 1: (a) An example image from the EIFFEL sequence. (b) The sparse 3D reconstruction result obtained by a conventional point-based SfM pipeline [4]. (c) The pointcloud combined with reconstructed 3D lines by our proposed method. On the right we can see an incrementally generated 3D mesh with (d) the 3D points only or (e) both points and lines. As we can see, the usage of complementary features significantly improves the completeness of the resulting 3D model in both cases.

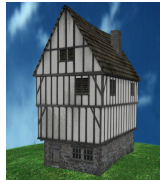

(a)

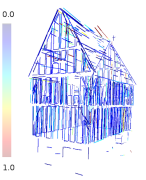

(b)

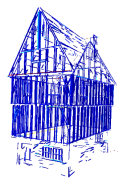

(c)

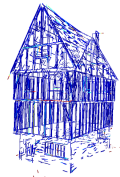

(d)

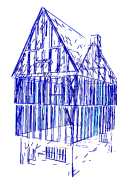

(e)
Figure 2: Reconstruction results for the Timberframe sequence (240 images). (a) Example image. (b) The original result by [6] (offline, runtime of several hours), RMSE $=0.291$. (c) The result by [2] (offline, runtime of 45 minutes), $\mathrm{RMSE}=0.094$. (d) The result by [3] (online, 5.7 minutes) RMSE $=0.196$. (e) Our reconstruction with appearance and collinearity constraints enabled (online, 6.9 minutes), RMSE $=0.095$.

\section{Results}

To demonstrate the capabilities of our proposed algorithm, we performed several quantitative and qualitative experimental evaluations. As a quantitative evaluation we used the synthetic Timberframe ${ }^{1}$ dataset from [6], since there is a groundtruth CAD model available. Figure 2 shows our result in comparison to related state-of-the-art methods [2, 3, 6].

As can be seen, our proposed method achieves more accurate results than a previous incremental approach [3] (RMSE 0.095 vs. 0.196), while the runtime is not largely increased (6.9 vs. $5.7 \mathrm{~min})$. That is off course due to the non-greedy nature of our approach and the incorporation of collinearity information. The accuracy with respect to the ground truth CAD model is almost as high as for the offline approach [2] (RMSE 0.095 vs. 0.094), which achieves the highest accuracy among the competitive algorithms, but with a significantly higher processing time (6.9 vs $45 \mathrm{~min}$ ). For more results, please see the full paper.

\section{Acknowledgements}

This work has been supported by the Austrian Research Promotion Agency (FFG) project FreeLine (843459) and OMICRON electronics GmbH.

[1] S. Agarwal, N. Snavely, I. Simon, and S.M. Seitz. Building rome in a day, 2009. International Conference on Computer Vision (ICCV).

[2] M. Hofer, A. Wendel, and H. Bischof. Line-based 3D reconstruction of wiry objects, 2013. Computer Vision Winter Workshop (CVWW).

[3] M. Hofer, A. Wendel, and H. Bischof. Incremental line-based 3D reconstruction using geometric constraints, 2013. British Machine Vision Conference (BMVC).

[4] C. Hoppe, M. Klopschitz, M. Rumpler, A. Wendel, S. Kluckner, H. Bischof, and G. Reitmayr. Online feedback for structure-frommotion image acquisition, 2012. British Machine Vision Conference (BMVC).

[5] C. Hoppe, M. Klopschitz, M. Donoser, and H. Bischof. Incremental surface extraction from sparse structure-from-motion point clouds, 2013. British Machine Vision Conference (BMVC).

[6] A. Jain, C. Kurz, T. Thormaehlen, and H. Seidel. Exploiting global connectivity constraints for reconstruction of 3D line segments from images, 2010. International Conference on Computer Vision and Pattern Recognition (CVPR).

[7] D. Lowe. Distinctive image features from scale-invariant keypoints, 2004. International Journal of Computer Vision (IJCV).

${ }^{1}$ http://www.mpi-inf.mpg.de/resources/LineReconstruction 